\section{AD's tangled tail}

\section{By Lev Osherovich, Senior Writer}

A Cleveland Clinic group has created a mouse model of Alzheimer's disease that bridges the mechanistic gap between two key disease-related proteins: amyloid- $\beta$ (A4) precursor protein and tau. ${ }^{1}$ The surprise is that the mice develop the disease phenotype independently of $\beta$-amyloid, the extracellular fragment of amyloid- $\beta$ (A4) precursor protein that many believe to be the prime suspect in Alzheimer's disease.

Instead, the disease in these mice appears to be caused by an intracellular fragment of amyloid- $\beta$ (A4) precursor protein (APP) that goes on to trigger both abnormal hyperphosphorylated microtubule-associated protein- $\tau$ (MAPT; FTDP-17; tau) phosphorylation and neuronal death-two hallmarks of human AD that are not seen in the most widely used mouse models.

The model thus raises fresh doubts about whether $\beta$-amyloid (A $\beta$ ) is really the cause of pathology and instead points to the intracellular portion of APP as the real culprit in AD.

$\mathrm{A} \beta$ is a byproduct of proteolytic processing of APP that accumulates on the outside of cells in amyloid plaques. $A \beta$ is toxic to neurons and is often detected in the serum and brains of $\mathrm{AD}$ patients.

Another characteristic feature of AD brains is the presence of intracellular tangles of tau.

Because commonly used mouse models of $\mathrm{AD}$ do not fully reproduce the abnormalities seen in the brains of $\mathrm{AD}$ patients, researchers have debated which of the two proteins is more important in pathology.

"The standard AD models aren't doing what they should do," said Donna Wilcock, assistant professor of neurology at Duke University School of Medicine.

Building on previous hints about the $A \beta$-independent effects of APP in cell culture, a team led by Sanjay Pimplikar, assistant staff member at the Cleveland Clinic, created knock-in mice that overexpress the intracellular domain of APP. These mice developed an ADlike syndrome without developing plaques, suggesting that plaques per se are not needed for disease.

After 18 months, mice overexpressing the APP intracellular domain had hippocampal neuron loss and modest memory deficits compared with controls. The transgenic mice also had high levels of hyperphosphorylated tau.

"The dominant hypothesis of $\mathrm{A} \beta$ as a causative agent may not be enough" to explain the paper's findings, said Pimplikar. "We have recapitulated most of the key features of the disease with just the intracel- lular APP fragment. These mice don't have any additional A $\beta$-this shows that you don't need A $\beta$ to have the disease."

Pimplikar's team also found that treating the mice with lithium chloride prevented memory problems compared with no treatment. Lithium inhibits glycogen synthase kinase $3 \beta$ (GSK3B), a kinase that phosphorylates tau. ${ }^{2}$

Pimplikar thus thinks the intracellular domain of APP activates GSK3B, which leads to tau phosphorylation and ultimately to neurodegeneration. He told SciBX that his findings could cut $A \beta$ out of the picture as a causative factor in $\mathrm{AD}$.

He also believes blocking the ability of the intracellular APP fragment to stimulate tau hyperphosphorylation may help halt AD progression.

The team is screening for small molecule modulators of APP's intracellular domain with the goal of reducing the activity of GSK3B and hopes to test leads in the mouse model. Pimplikar has sought patents on a screening procedure to identify such compounds.

\section{Model mouse}

Pimplikar's hypothesis does not fully explain earlier results with other mouse models of AD. For example, mice that overexpress full-length App develop A $\beta$ plaques but do not build up hyperphosphorylated tau or undergo neurodegeneration.

Wilcock told SciBX it was unclear why mice that overexpress full-length App do not also have increased levels of the intracellular domain and accompanying neurological abnormalities similar to those seen in Pimplikar's mice.

Moreover, it is not known whether the intracellular domain is sufficient for the full spectrum of abnormalities seen in AD. Pimplikar's team did not report whether the hyperphosphorylated tau in the mice went on to form neurofibrillary tangles-the intracellular aggregates of tau protein that nearly always accompany AD.

"There's a change in tau and some loss of hippocampal neurons, but there are no plaques or tangles" with the Cleveland Clinic mice, said Dale Schenk, CSO of Elan Corp. plc.

He said he would reserve judgment on whether "this intracellular fragment is relevant" until evidence of the intracellular domain's role in human disease is shored up. "What I would like to see is a more thorough analysis of other areas in the brain such as the cortex," Schenk told SciBX.

Schenk added that the overexpression system used in the study could make intracellular APP fragments "go to places or do things that never happen in nature." He said further physiological studies are needed to determine whether the pathology caused by the intracellular domain is really a good model for AD.

Pimplikar noted that brain lysates from $\mathrm{AD}$ patients have higher levels of the APP intracellular domain than those from healthy controls, thus suggesting the fragment has a role in disease. 


\section{TARGETS \& MECHANISMS}

\section{Tau of AD}

If Pimplikar's hypothesis that the intracellular domain of APP sets off a cascade that leads to AD pathogenesis proves correct, it could lend additional validation to therapeutics that block production of the APP intracellular fragment or molecules that target tau.

Both $A \beta$ and the intracellular fragment are generated by the action of $\gamma$-secretase and $\beta$-site APP-cleaving enzyme 1 (BACE1). Thus, inhibitors of these enzymes could hit two birds with one stone-blocking the production of both $A \beta$ and the intracellular domain.

The most advanced $\gamma$-secretase inhibitor, semagacestat (LY450139) from Eli Lilly and Co., is in Phase III testing for AD. The most advanced BACE1 inhibitor, CTS-2116 from CoMentis Inc. and Astellas Pharma Inc., is in Phase I testing for AD.

Pimplikar's paper is also "very supportive of the tau hypothesis," said J. Steven Whitaker, CMO of Allon Therapeutics Inc. "We've always thought that in AD pathophysiology, tau hyperphosphorylation is having an injurious effect on the neurons."

Allon's davunetide (AL-108), a neuroprotective peptide that binds to tau, has completed Phase IIa trials for mild cognitive impairment, a precursor to AD. This fall, the compound is expected to start a Phase III trial for frontotemporal dementia (FTD), another taurelated neurodegenerative condition.

According to Whitaker, targeting tau has a disease-modifying effect in animal models of AD and FTD, but the strategy has been overshadowed by efforts to deplete $\mathrm{A} \beta$. He said that in another mouse model of tau pathology, davunetide caused "a dramatically significant reduction of tau hyperphosphorylation, about $75 \%$ compared with controls," and that this decrease led to behavioral improvements.

Whitaker said the main novelty of Pimplikar's study is the link between alterations in APP processing and tau pathology, although he wanted to see further mechanistic studies to establish exactly how the intracellular APP fragment leads to tau phosphorylation.

The findings in the paper, published in the Proceedings of the National Academy of Sciences, have not been patented.

Osherovich, L. SciBX 2(43); doi:10.1038/scibx.2009.1591

Published online Nov. 5, 2009

\section{REFERENCES}

1. Ghosal, K. et al. J. Proc. Natl. Acad. Sci. USA; published online Oct. 15, 2009; doi:10.1073/pnas.0907652106

Contact: Sanjay W. Pimplikar, Cleveland Clinic, Cleveland, Ohio e-mail: pimplis@ccf.org

2. Ryan, K.A. et al. J. Cell Biol. 171, 327-335 (2005)

\section{COMPANIES AND INSTITUTIONS MENTIONED}

Allon Therapeutics Inc. (TSX:NPC), Vancouver, British Columbia, Canada Astellas Pharma Inc. (Tokyo:4503), Tokyo, Japan

Cleveland Clinic, Cleveland, Ohio

CoMentis Inc., South San Francisco, Calif.

Duke University School of Medicine, Durham, N.C.

Elan Corp. plc (NYSE:ELN), Dublin, Ireland

Eli Lilly and Co. (NYSE:LLY), Indianapolis, Ind. 